Arts

et Savoirs

\section{Arts et Savoirs}

$14 \mid 2020$

Styles de pensée, pensées du style. Écrire le vivant au XIXe siècle

\title{
Style et métaphores naturalistes : le projet renanien d'une embryogénie de l'esprit humain
}

Naturalistic style and metaphors: Ernest Renan's project of an embryogenesis of the human spirit

\section{Azélie Fayolle}

\section{OpenEdition}

\section{Journals}

Édition électronique

URL : http://journals.openedition.org/aes/3211

DOI : 10.4000/aes.3211

ISSN : 2258-093X

Éditeur

Laboratoire LISAA

Référence électronique

Azélie Fayolle, "Style et métaphores naturalistes : le projet renanien d'une embryogénie de l'esprit humain », Arts et Savoirs [En ligne], 14 | 2020, mis en ligne le 29 décembre 2020, consulté le 21 février 2021. URL : http://journals.openedition.org/aes/3211 ; DOI : https://doi.org/10.4000/aes.3211

Ce document a été généré automatiquement le 21 février 2021.

Centre de recherche LISAA (Littératures SAvoirs et Arts) 


\section{Style et métaphores naturalistes : le projet renanien d'une embryogénie de l'esprit humain}

Naturalistic style and metaphors: Ernest Renan's project of an embryogenesis of the human spirit

\section{Azélie Fayolle}

\section{NOTE DE L'AUTEUR}

Une première version de cet article a été présentée au Congrès de la Nineteenth-Century French Studies (NCFS) à Charlottesville en novembre 2017.

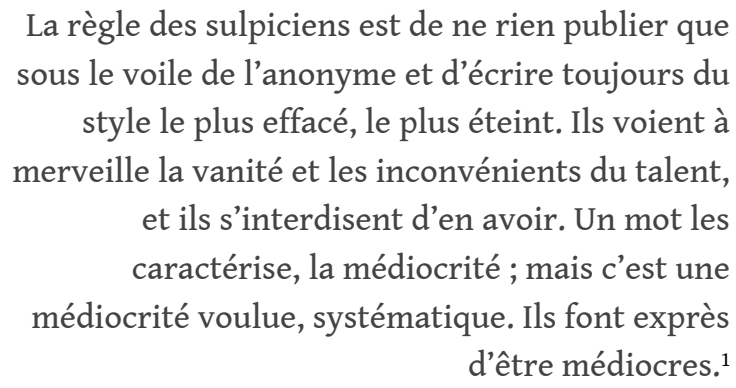

Par ses publications comme par sa vie savante, Renan est progressivement devenu, au fil du siècle, le symbole vivant ${ }^{2}$ de la lutte de la science contre la foi et l'obscurantisme, au point d'être une figure tutélaire de la Troisième République. Il faut dire que la publication de la Vie de Jésus en 1863 avait mis le feu aux poudres, après les inquiétudes déjà suscitées par De l'Origine du langage $(1848)^{3}$ et la nomination, en 1862, d'un laïc, en outre défroqué, à la chaire d'études hébraïques du Collège de France. Best-seller (et longseller) européen, aujourd'hui toujours publié en poche, la Vie de Jésus a valu à Renan le titre de "blasphémateur européen", octroyé par le Pape Pie IX ${ }^{4}$ l'Angélus pour la messe de Pâques, des milliers de lettres, d'insultes et de libelles, ainsi que sa révocation 
du Collège de France ${ }^{5}$. Renan se défend pourtant d'avoir voulu produire un ouvrage scandaleux, en répondant à la polémique comme à la controverse dans sa Préface de la treizième édition (1867). S'il est certain qu'il y a dans ses affirmations une part de stratégie médiatique ${ }^{6}$, la Vie de Jésus inaugure pourtant un projet longuement médité : la somme qu'est l'Histoire des origines du christianisme (1863-1883). Les premiers linéaments de ce projet se trouvent dès les Cahiers de jeunesse, rédigés au séminaire de Saint-Sulpice dans les années 1845-1846. C'est en effet pendant ces années que Renan en esquisse les premières bases, en parallèle de son traité de grammaire hébraïque (couronné du prix Volney en 1847). L'Histoire des origines $d u$ christianisme, que prolongera une tout aussi monumentale Histoire du peuple d'Israël (1887-1893), réalise alors le projet ancien de fonder une embryogénie de l'esprit humain. La métaphore, filée dans ses œuvres, est l'indice le plus repérable d'un modèle de pensée qui structure l'ensemble des travaux historiens de Renan. La récurrence de cette formule métaphorique signale la permanence du modèle de pensée embryologique, utilisé par Renan comme matrice d'une philosophie de l'histoire et comme inspiration méthodologique. Il s'agit en effet pour l'historien de remonter aux « origines obscures » du langage et de la religion. Les métaphores issues des sciences naturelles qui émaillent les textes de Renan n'ont ainsi rien d'ornemental : elles constituent la partie émergée d'un modèle de pensée difficile à percevoir pour la plupart des lecteurs de Renan, et dont il faut restituer le fonctionnement. Renan promeut une lecture laïque et scientifique des Évangiles : en récusant la Révélation, il ouvre la voie à une approche nouvelle des textes en France. La Vie de Jésus ne se présente pas comme une réponse brutale et anticléricale aux Évangiles: le livre se place au contraire au plus près des textes néo-testamentaires, au point de s'approcher, à plusieurs reprises, d'une réécriture proche de la citation in extenso. Renan se situe à la croisée des Évangiles et des sciences naturelles, par une remotivation sémantique du texte biblique chargée de connotations naturalistes. Grâce à ce choix stylistique, Renan met en place une pensée du texte et du document qui trouve son assise dans le présupposé d'un texte pensé comme organique.

\section{Projet d'une embryogénie de l'esprit humain}

2 Si Darwin ne publie son Origin of Species qu'en $1859^{7}$, Renan a pu lire très jeune quelques présentations des idées de Lamarck, notamment dans le Musée des familles, un périodique auquel il était abonné l'année de ses douze ans ${ }^{8}$. L'idée d'une nature en mouvement, esquissée dans les leçons d'histoire naturelle de Pierre Boitard que Renan peut alors découvrir, se retrouvera dans les cours de philosophie et de sciences du séminaire comme, paradoxalement, d'hébreu : à l'instar de Darwin et d'abord sans le connaître, l'évolution des langues permet à Renan de supposer une forme, assez vague, d'évolution des espèces ${ }^{9}$. Il postule l'idée d'une nature en mouvement, pensée comme un éternel fieri téléologique ${ }^{10}$. Cette idée d'une nature en mouvement, visible par une transformation des espèces, gagne en importance dans la première moitié du $\mathrm{XIX}^{\mathrm{e}}$ siècle, en particulier lors de la querelle des analogues qui oppose Cuvier à Geoffroy Saint-Hilaire, querelle dont Goethe fait un compte rendu et qui a un écho phénoménal ${ }^{11}$. La possibilité du transformisme, suggérée par l'unité de plan théorisée par Geoffroy Saint-Hilaire ${ }^{12}$, repose sur les sciences tératologiques et embryologiques que ce dernier fonde : l'étude du développement des êtres vivants se situe au cœur de la 
biologie naissante. Avec l'appui de ses cours à Saint-Sulpice, et notamment de ceux de physique et de sciences naturelles délivrés par l'abbé Pinault, les sciences naturelles s'imposent progressivement pour Renan comme un modèle dominant qui le conduit à élaborer, par tâtonnements successifs, son projet d'embryogénie de l'esprit humain développé dans son ouvrage programmatique, L'Avenir de la science $(1848,1890)$ :

Or, de même qu'à côté de la science des organes et de leurs opérations, il y en a une autre qui embrasse l'histoire de leur formation et de leur développement, de même à côté de la psychologie qui décrit et classifie les phénomènes et les fonctions de l'âme, il y aurait une embryogénie de l'esprit humain, qui étudierait l'apparition et le premier exercice de ces facultés dont l'action, maintenant si régulière, nous fait presque oublier qu'elles n'ont été d'abord que rudimentaires. ${ }^{13}$

3 Le parallèle entre les sciences naturelles et les sciences de l'esprit humain, que Renan contribue à fonder en sciences humaines ${ }^{14}$, est appuyé ; la similitude de méthode n'est pas fortuite, mais elle révèle comment l'historien pense l'histoire à l'instar d'une anatomie historique (« la science des organes et de leurs opérations » accompagnée de la science de "l'histoire de leur formation et de leur développement", périphrase désignant l'embryologie), en postulant une psychologie historique - autrement dit, l'histoire et les sciences sont unifiées et ne se distinguent que par l'adaptation à leurs objets d'étude. Le parallélisme n'en est un qu'en apparence: il n'y a pas, dans la perspective renanienne, de véritable saut logique à penser une science naturelle appliquée à ce qu'il appelle les "produits de l'esprit humain », dans la mesure où c'est une même science qui embrasse les disciplines particulières, d'abord définies par leur objet d'étude. Cette discipline nouvelle, pour laquelle Renan invente la formule d'embryogénie de l'esprit humain, est ensuite explicitée :

Une telle science serait sans doute plus difficile et plus hypothétique que celle qui se borne à constater l'état présent de la conscience. Toutefois il est des moyens sûrs qui peuvent nous conduire de l'actuel au primitif, et, si l'expérimentation directe de ce dernier état nous est impossible, l'induction s'exerçant sur le présent peut nous faire remonter à l'état qui l'a précédé et dont il n'est que l'épanouissement. En effet, si l'état primitif a disparu pour jamais, les phénomènes qui le caractérisaient ont encore chez nous leurs analogues. Chaque individu parcourt à son tour la ligne qu'a suivie l'humanité tout entière, et la série des développements de l'esprit humain est exactement parallèle au progrès de la raison individuelle, à la vieillesse près, qu'ignorera toujours l'humanité, destinée à refleurir à jamais d'une éternelle jeunesse. ${ }^{15}$

Renan part ici de l'hypothèse d'une évolution récapitulative ${ }^{16}$, théorie qui sera amplement diffusée par Haeckel dans la seconde moitié du xIX ${ }^{e}$ siècle, selon laquelle l'évolution de l'individu suit les mêmes étapes que l'évolution de l'espèce à laquelle il appartient. S'y ajoute l'hypothèse d'une marche différenciée des peuples, qui permet des études comparées, en supposant un développement uniforme pour toutes les espèces, mais à la vitesse et à l'aboutissement différents. La similitude des lois de développement entre produits naturels et produits de l'esprit humain permet à l'historien de recourir à des méthodes déjà éprouvées par les sciences naturelles et la philologie: l'induction et l'analogie. La formule qui résume le projet renanien d'élaborer une "embryogénie de l'esprit humain " est ainsi sentie comme métaphorique (comme le marque l'italique), tout en ne reposant pas sur un saut logique : c'est en s'appuyant sur la croyance en différents âges de l'humanité, ramenée à un grand individu spirituel ("l'esprit humain»), que Renan peut remonter, en conjuguant analogie et induction, aux temps des origines, temps qu'il qualifie d'« embryonnaires ». La métaphore des "âges de l'humanité » est topique; Renan la vivifie et la motive 
scientifiquement en remontant au développement embryonnaire, terme à la connotation plus technique que le terme plus quotidien "enfance " qu'il s'agit de dépasser. Le projet d'une embryogénie de l'esprit humain trouve ainsi son socle dans une pensée globale de la science et de ses divisions disciplinaires.

\section{Embryogénie : histoire d'une métaphore renanienne}

5 Les premiers modèles de pensée de Renan sont fortement inspirés par son goût précoce pour les mathématiques et les cours de physique de l'abbé Pinault suivis au séminaire de Saint-Sulpice, dans l'annexe d'Issy (1841-1843). Le passage d'un modèle physique à un modèle issu explicitement des sciences naturelles a certainement été encouragé par les cours de sciences reçus au séminaire ; leur assimilation a cependant été progressive. Si l'élaboration de ce modèle de pensée a été lente, c'est bien un intérêt ancien que l'on retrouve sous la plume du jeune séminariste au tournant de 1843-1844, qui peut se plaire à imaginer les origines embryonnaires du Christ lui-même :

On considère d'ordinaire Notre-Seigneur à la crèche et sur la croix, quand on veut se former une idée de ses humiliations : mais il $\mathrm{y}$ a une position où j'aime bien mieux l'envisager sous ce rapport. C'est dans le sein de Marie, à l'état d'embryon. Je voudrais que le système des animaux spermatiques fût vrai pour pouvoir l'envisager sous une forme plus humble, et dire à la lettre: Vermis sum et non homo. ${ }^{17}$

6 Dans cette rêverie christique, il s'agit pour le jeune séminariste de reprendre le verset 7 du psaume 22 (21 dans la numérotation grecque) ${ }^{18}$ et de le lire littéralement par un petit exercice d'imagination, conduisant à la rencontre entre le verset biblique et la théorie des animaux spermatiques, dont l'existence n'est pas encore démontrée. Sous la plume du jeune Renan, ce ver (ou insecte, selon les traditions exégétiques) devient " embryon», voire, à un stade antérieur, spermatozoïde ${ }^{19}$. La question, largement débattue par l'exégèse biblique, de la nature ou de la substance du Christ est ramenée par Renan aux théories naturalistes débattues à son époque. Les occurrences du terme « embryon » ou de ses dérivés restent cependant assez peu présentes dans les écrits du séminariste, même dans ses Cahiers de jeunesse, où on n'en trouve que de furtives apparition $s^{20}$. On retrouve le mot dans les premiers textes publiés de Renan, comme De l'Origine du langage (1848) et Histoire générale et système comparé des langues sémitiques (prix Volney 1847, 1855). L'idée d'un état embryonnaire apparaît dès 1848 :

On ne peut trop soigneusement distinguer, quand il s'agit des langues, l'état embryonnaire, durant lequel des accidents indifférents à l'âge adulte ont pu avoir une importance capitale, de l'état parfait, où elles se sont fixées, pour ainsi dire, dans un moule définitif. L'état embryonnaire des langues a pu durer fort peu de temps; mais il a existé, et à ce moment, où se formait l'individualité des races, la nature humaine, encore flexible, a dû recevoir pour l'éternité des traces profondes. On peut dire avec vérité que le sort de chaque être se détermine dans le sein de sa mère, de même que, sur le sommet des montagnes, au point où se fait la séparation des eaux, un pli de terrain décide du cours des plus grands fleuves, et les prédestine à porter leurs eaux à telle ou telle mer. ${ }^{21}$

7 L'image de l'embryon est singulièrement riche ${ }^{22}$ : elle évoque une langue vivante, à l'apparition spontanée, ce qui entérine l'idée de langues pensées comme des organismes et de familles de langues, dans la ligne des théories linguistiques des romantiques allemands et en particulier de Jacob Grimm, cité dans la préface de De l'Origine du langage. Se dégage ici une caractéristique du style renanien: la reprise de 
formules connues, voire topiques, se fait en leur apportant une remotivation sémantique, souvent marquée par une charge scientifique nouvelle, et une extension élargie.

Si le modèle de l'embryon a l'avantage d'être particulièrement imagé, il est également riche de significations et de connotations qu'il faut préciser. L'embryon suppose un certain anthropomorphisme, ou du moins un organicisme, qui révèle l'inscription dans le temps de ce modèle de pensée naturaliste : l'embryon est voué à se développer. Ce modèle embryologique sous-entend en effet une vie de l'organisme et un développement déjà prédéterminé par la structure interne de l'embryon - que Renan nomme assez classiquement "germe ». Le germe est dans ses travaux la principale image permettant de désigner l'origine, l'apparition et la diffusion des langues ou des religions ; il prend la suite des concepts de «moule » et de " casier », présents dans les premiers textes linguistiques de Renan : le modèle organiciste remplace les métaphores mécanicistes.

Le terme de "germe" a un grand avantage : il est d'une extension particulièrement large. Son premier sens est quasi botanique : le germe désigne la " partie de la semence qui se développe en formant la plante", dans un sens courant bien plus que spécifiquement scientifique ${ }^{23}$. Le germe est la première pousse issue de la graine et il peut, par extension, désigner la graine elle-même. L'Histoire générale et système comparé des langues sémitiques montre l'avantage du " germe » sur le "moule ", qui n'est qu'un cadre extérieur, alors que le germe suppose une détermination intérieure :

Il faut admettre dans l'univers ce qui se remarque dans la plante et l'animal, une force intime qui porte le germe à remplir un cadre tracé d'avance. Il y a une conscience obscure de l'univers qui tend à se faire, un secret ressort qui pousse le possible à exister. L'être m'apparaît ainsi comme un compromis entre des conditions opposées ; comme une équation qui, dans la plupart des hypothèses, donne des solutions négatives ou imaginaires, mais qui, dans certains cas, en donne de réelles ; comme un van qui ne laisse passer que ce qui a droit de vivre, c'est-àdire ce qui est harmonieux..$^{24}$

10 Le germe reprend ainsi l'idée d'une détermination interne; il fait passer le conditionnement des produits de l'esprit humain du côté des êtres naturels, en entérinant l'organicisme des objets artificiels. Comme l'embryon, il suppose en outre un polygénisme linguistique : les langues peuvent être sœurs, cousines, mais elles ne dérivent pas toutes d'une source unique ${ }^{25}$.

11 Le germe est transformé par Renan en matrice d'une métaphore filée qui structure et unifie la plus grande partie de ses travaux. L'image trouve cependant une motivation nouvelle à partir des années 1860 , c'est-à-dire aux débuts de l'Histoire des origines $d u$ christianisme : le germe n'est plus seulement compris comme une métaphore botanique, mais également comme un microorganisme, voire un virus. La conversion du " germe " de la botanique en virus se manifeste par plusieurs points. Le pluriel, s'il était présent, devient plus fréquent : il marque la prise en compte de réalités concrètes, au contraire du singulier, plus abstrait. C'est au contact des métaphores épidémiologiques que le germe trouve son sens viral : la compréhension de la contamination connait, grâce aux travaux de Pasteur, d'importants progrès. Le germe se charge ainsi dans les études d'histoire religieuse d'une connotation scientifiquement plus précise: de botanique, mais visible, le germe en vient à désigner l'invisible. Il acquiert alors une connotation potentiellement négative, soulignée par la métaphore épidémiologique : la ferveur, désignée comme fièvre, se prolonge dans la contagion. 
Le choix de ces termes montre comment Renan fait se rencontrer sciences naturelles et étude de l'histoire des religions. C'est en effet la circulation des idées et des mentalités qui l'intéresse. Contrairement à des textes comme Averroès (1852) ou l'Histoire de l'étude de la langue grecque $(1847,2009)$, qui montrent des transferts épistémologiques et des habitudes savantes ${ }^{26}$, les études religieuses s'appuient sur des métaphores médicales : le sentiment religieux obéit ainsi pour lui à un modèle épidémiologique. Parce que la religion est un produit de l'esprit humain dont le développement obéit aux lois de l'organisme, et parce qu'elle est le produit de corps caractérisés par une physiologie particulière - notamment perceptible dans la pratique du jeûne ascétique -, elle peut se traduire par un modèle de pensée organiciste. Ce qui distingue la religion, comme produit de l'esprit humain, des productions intellectuelles, comme les traditions savantes, c'est qu'elle met en jeu les émotions - la ferveur - des corps qu'elle convertit : la religion se trouve au croisement de l'histoire intellectuelle et d'une histoire du sentiment, qui est alors encore à écrire.

\section{La Bible et le savant}

13 Le projet de fonder une embryogénie de l'esprit humain repose donc, dès ses premières esquisses, sur la prise en compte littérale d'une métaphore: l'image dicte la méthodologie de travail; elle signale le modèle de pensée. La différence entre « métaphore » et « modèle de pensée » a été établie par Max Black en 1962 : le modèle de pensée repose sur un développement théorique, quand la métaphore a une fonction plus ornementale ${ }^{27}$. L'embryogénie n'est ainsi pour Renan pas une simple ornementation : elle se fait guide méthodologique. De l'embryon initial, qui constitue le nouvel objet de l'historien, sont issues d'autres métaphores qui soutiennent et prolongent le modèle de pensée. Renan s'intéresse en effet aux temps des origines, qualifiés d'embryonnaires, comme aux différentes modalités du développement intellectuel, par la diffusion des idées et de l'érudition comme des religions. C'est ainsi qu'à partir d'un germe initial se propage une contagion chrétienne: le modèle épidémiologique lui permet de décrire la diffusion du christianisme. L'Histoire des origines $d u$ christianisme repose sur un réseau serré de métaphores naturalistes qui assimilent l'étude de l'histoire religieuse à une nosologie. Il est alors frappant de constater que ces métaphores ont une base religieuse : la ferveur ouvre à la fièvre, la diffusion est une contamination et une propagation, reposant sur un germe originel.

14 Ce germe s'inscrit dans un continuum tissé par Renan: de la parabole du germe de blé des Évangiles au germe viral, Renan reprend les motifs et les images bibliques pour les remotiver dans un sens scientifique et naturaliste :

Le germe de cette grande révolution sera d'abord méconnaissable. Il sera comme le grain de sénevé, qui est la plus petite des semences, mais qui, jeté en terre, se change en un arbre sous le feuillage duquel les oiseaux viennent se reposer; ou bien il sera comme le levain qui, déposé dans la pâte, la fait fermenter toute entière. 28

Ce passage reprend quasi littéralement le texte des Évangiles, en particulier Matthieu :

Jésus leur proposa une autre parabole, et il dit: le royaume des cieux est semblable à un grain de sénevé qu'un homme a pris et semé dans son champ. C'est la plus petite de toutes les semences ; mais, quand il a poussé, il est plus grand que les légumes et devient un arbre, de sorte que les oiseaux du ciel viennent habiter dans ses branches. ${ }^{29}$ 
La comparaison de ces deux textes nous permet de voir la fabrique du style de Renan : les textes bibliques, longuement médités, coulent pour ainsi dire sous sa plume. La phrase précédant la reprise biblique oriente néanmoins la citation: le "germe " joue ainsi un rôle de pivot entre la parabole et le virus nouvellement découvert. Le terme n'active pas alors les connotations morbides qu'il peut avoir aujourd'hui, mais il permet surtout, dans ce cotexte, de manifester la contagion virale, tout en inscrivant la Vie de Jésus dans une longue filiation historique. Les métaphores de la contagion prolongent la métaphore germinale; elles sont elles aussi des réécritures du fait religieux. Se comprend alors une autre caractéristique du style renanien : l'Histoire des origines du christianisme, et en particulier la Vie de Jésus, sont des réécritures scientifiques (et sélectives) de la Bible. Il ne s'agit pas d'introduire de nouveaux termes dont Renan, après quelques tentatives néologiques dans ses Cahiers de jeunesse, se défie, mais de garder les mêmes termes, en leur injectant une connotation scientifique (et notamment virale) nouvelle. Les règles sulpiciennes se retrouvent jusque dans la scandaleuse Vie de Jésus : au style « éteint », entretenu par Renan, répond la reprise quasi littérale, mais transformée, du texte évangélique - le feu couve sous la cendre. Les mêmes mots se trouvent, non vidés de leur sens, mais réorientés : la remotivation n'annule pas les connotations précédentes, mais elle s'y ajoute. Le mouvement de l'histoire telle que le pense Renan se retrouve dans son style : les formes anciennes, voire antiques de la langue peuvent être remotivées scientifiquement, comme les institutions du passé que sont les religions finiront, si l'on suit le programme de L'Avenir de la science, par être justifiées et prouvées par la science.

\section{Vers le texte organisme}

18 La remotivation stylistique opérée par Renan par cette réécriture des Évangiles s'appuie sur une longue méditation (rumination, en ses propres termes) lexicale et philologique. Le style renanien, style parfois qualifié par ses détracteurs de « mol » ou d'« efféminé $"^{30}$, se caractérise par une phrase insaisissable, et est qualifié d'« éteint " ou d'" adouci " par Renan lui-même. C'est notamment par la reprise des termes et des formules les plus classiques que se joue ce paradoxe d'une écriture subversive et dans le même temps sans accroc. Si l'affirmation d'un style "éteint » est quelque peu à nuancer pour les textes de Renan ${ }^{31}$, il reste que ces particularités stylistiques sont le fruit d'un projet épistémologique plus vaste, qui affleure dans le texte ?) par la métaphore - en réalité modèle de pensée, dont le soubassement théorique reste inaccessible à la majorité des lecteurs ${ }^{32}$. Le style de Renan traduit son projet épistémologique; il s'appuie dans le même temps sur une pensée nouvelle du document.

Renan est en effet le premier, en France ${ }^{33}$, à considérer les textes sacrés comme des textes historiques : il s'agit alors de dégager la légende pour retrouver, sinon les faits vrais, du moins les plus probables de la biographie du Christ (dans la Vie de Jésus) et de la diffusion du christianisme (dans la suite de la somme historique qu'est l'Histoire des origines $d u$ christianisme). Autrement dit, les Évangiles peuvent être étudiés scientifiquement comme le sont les textes fondateurs d'autres religions, ou plus largement l'ensemble des textes. La sécularisation du sacré commence par cet acte transgressif, rendu plus subversif par le statut de laïc de Renan et par sa livraison à un public de non-spécialistes ${ }^{34}$. Cette réécriture scientifique de la Bible appelle à une 
relecture critique par le public, alors que la pratique de la lecture personnelle reste, il faut le rappeler, peu encouragée dans un contexte catholique, à un moment où la messe se déroule encore dos au public et en latin. Cet élargissement du corpus des documents étudiés par les philologues (et, potentiellement, par le public) n'est pas seulement textuel : il se manifeste aussi par la pluridisciplinarité qu'appelle de ses vœux Renan dès l'élaboration de son modèle méthodologique. L'élargissement du corpus et des méthodes est aussi naturaliste : les documents étudiés ne s'inscrivent pas seulement sur le papier ou sur la pierre. Ainsi Renan a-t-il pu se renseigner auprès de géologues et de botanistes historiques pour imaginer le paysage galiléen traversé par Jésus, paysage que l'historien parcourt lui-même :

J'eus devant les yeux un cinquième Évangile, lacéré, mais lisible encore, et désormais, à travers les récits de Matthieu et de Marc, au lieu d'un être abstrait, qu'on dirait n'avoir jamais existé, je vis une admirable figure humaine vivre, se mouvoir..$^{35}$

Renan procède ainsi à un élargissement de la notion de document : le document n'est plus exclusivement textuel, mais il peut se faire matériel, esthétique, jusqu'au paysage, qui acquiert le même statut documentaire qu'un texte. En considérant les religions ou les langues comme des organismes, Renan donnait déjà une extension élargie à ce qui fait document : celui-ci peut devenir un territoire, une vision, dont l'analyse est étayée par les différentes sciences. La prise en compte du paysage ne permet pas seulement une attestation d'authenticité de l'historien par un témoignage quasi direct (distance temporelle mise à part), mais elle consiste aussi en une forme de renouvellement de la théorie des climats de Montesquieu (très peu cité par Renan), non par une prétendue influence des températures, mais en supposant une imprégnation par le spectacle des paysages. Si la figure, problématique, du Sémite marqué par la vie sous la tente dans le désert provient de la même vision historienne, celle de Jésus influencé par les rives du lac de Génésareth conduit à l'écriture de la Vie de Jésus comme une " pastorale »" ${ }^{36}$ et à une polarisation forcée entre Nazareth et Jérusalem.

21 Cet élargissement de la notion de document s'accompagne d'une réflexion sur le texte produit : ce dernier fait l'objet d'une attention stylistique scrupuleuse, soutenue par de nombreuses réflexions sur l'écriture, éparses dans les différents travaux de Renan ${ }^{37}$. C'est encore l'organisme qui sert de modèle, non de pensée, mais ici d'écriture : «Ce sentiment d'un organisme vivant, on n'a pas hésité à le prendre pour guide dans l'agencement général du récit ", écrit Renan dans sa préface à la treizième édition de la Vie de Jésus (1867). Une " part de divination et de conjecture » est alors, selon lui, permise :

Une grande vie est un tout organique qui ne peut se rendre par la simple agglomération de petits faits. Il faut qu'un sentiment profond embrasse l'ensemble et en fasse l'unité. La raison d'art en pareil sujet est un bon guide; le tact exquis d'un Goethe trouvait à s'y appliquer. La condition essentielle des créations de l'art est de former un système vivant dont toutes les parties s'appellent et se commandent. Dans les histoires du genre de celle-ci, le grand signe qu'on tient le vrai est d'avoir réussi à combiner les textes d'une façon qui constitue un récit logique, vraisemblable, où rien ne détonne..$^{38}$

Les organismes ne guident plus seulement l'historien pour l'analyse des textes, mais pour la production du texte historien lui-même : le "sentiment profond", celui de l'historien qui doit se transmettre ensuite au lecteur, conditionne la composition et le style de l'ouvrage. Il ne s'agit pas alors d'harmonie, mais de la transposition moderne de cet idéal classique : l'organisme permet de se couler dans le modèle rhétorique du 
Grand Siècle (tout sulpicien), tout en le chargeant de connotations scientifiques. Le texte de l'historien doit ainsi se faire, à l'image de l'objet étudié, organique, c'est-à-dire donner une impression de (grande) "vie», tout en étant cohérent et uni. Le «sentiment profond", qui préside à la conception de l'ouvrage historien, conjoint à l'unité de l'ensemble, n'est pas sans rappeler l'unité de plan théorisée par Geoffroy Saint-Hilaire : le sentiment profond, en donnant son sens à l'ensemble, oriente "les petits faits " pour les structurer en un récit - et la vie du Christ dont il s'agit de faire la biographie se pense comme celle d'un corps, et doit avoir eu un déroulement lui aussi organique. Cette écriture de l'organisme rejoint alors la vision programmatique élaborée dans L'Avenir de la science: cette vie qu'il faut retrouver en écrivant une monographie sur Jésus s'insère elle-même dans un ensemble plus vaste, pour construire l'édifice séculaire de la science, dont l'évolution est, elle aussi, guidée par les lois de la nature.

\section{Conclusion}

Le style renanien se distingue par sa simplicité apparente et sa facture classique: les rares métaphores qui le parsèment et la fluidité de sa phrase l'opposent aux ruptures syntaxiques d'un Michelet. La souplesse de son style laisse le sujet traité au premier plan du texte ; les métaphores issues des sciences naturelles signalent la scientificité de la réécriture qui parachève l'étude des textes sacrés menée par Renan. Son écriture n'use pas des sciences naturelles par souci d'ornementation, mais par un élargissement des objets d'étude et des méthodes de l'histoire : tout, pour lui, devient objet de nature et donc objet d'histoire, l'histoire jouant elle-même le rôle de conscience réflexive d'une nature toujours en mouvement.

L'idée même de style devient difficile à maintenir, dans ce contexte à maintenir : point de coquetterie pour Renan, pour lequel l'esthétique révèle toujours une épistémologie. Les métaphores ne peuvent pas, en l'absence de développement théorique explicatif au sein des textes dans lesquels elles apparaissent, se définir comme de véritables modèles de pensée tels que les définit Max Black; placées dans un véritable entre-deux définitionnel, les métaphores renaniennes nécessitent une redéfinition de leur situation textuelle, au sein de l'ensemble de l'œuvre de Renan. Reste alors (au moins) une question: pourquoi Renan n'a-t-il pas explicité l'étayage théorique de ces métaphores? Probablement pour hâter la réunion de la science et de la foi professée dans L'Avenir de la science, sans aiguiser davantage la réflexivité de l'âge critique. L'historien s'efface alors dans son texte pour laisser place à l'histoire.

\section{NOTES}

1. Ernest Renan, Souvenirs d'enfance et de jeunesse, 1883, CEuvres complètes, éd. d'Henriette Psichari, Paris, Calmann-Lévy, t. II, 1947-1961, p. 830. Toutes les citations de Renan proviennent de cette édition. 
2. Voir, à titre d'exemple significatif, les caricatures faites de Renan, dans mon article Renan caricaturé : traits, formes et pleins du savant, Études renaniennes, $\mathrm{n}^{\circ}$ 118-119, 2018, p. 11-47.

3. Déjà mis à l'index, voir Jean-Baptiste Amadieu, «Renan jugé par la Congrégation de l'Index », Études Renaniennes, $\mathrm{n}^{\circ}$ 113, 2012, p. 97-121.

4. D'après Jean Balcou, l'appellation date du numéro de L'Osservatore romano du 29 octobre 1872 , «Pie IX vu par Renan », dans Henry Laurens (dir.), La science, la religion, la République, Paris, Odile Jacob, « Travaux du Collège de France », 2013, p. 290, note 14.

5. Renan a lui-même rassemblé les pièces de sa défense en plaquette puis dans ses Questions contemporaines (1868).

6. Scandale néanmoins habilement attisé par Renan, voir Nathalie Richard, La Vie de Jésus de Renan, la fabrique d'un best-seller, Rennes, Presses Universitaires de Rennes, « Histoire », 2015.

7. L'ouvrage n'est traduit en français qu'en 1862 par Clémence Royer. Il ne figure pas dans la bibliothèque de Renan.

8. Au détour d'une lettre du 29 septembre 1836 de la sœur d'Ernest Renan, Henriette, à leur mère, on trouve la proposition de prolonger l'abonnement d'Ernest au Musée des familles, qui « expire ce $1^{\mathrm{er}}$ octobre ", Ernest Renan, Jean Balcou (dir.) Correspondance générale, Paris, Honoré Champion, «Textes de littérature moderne et contemporaine", vol. I, 1995, p. 29. On trouve dans le Musée des familles de juin 1836 une leçon naturelle transformiste du naturaliste Pierre Boitard, "Paris avant les hommes ": il s'agit de la première version de son roman préhistorique, publié (à titre posthume) en 1861.

9. Notamment dans De l'Origine du langage (1848), dans lequel il appelle à des influences réciproques entre philologie et anatomie.

10. Voir notamment Keith Gore, L'Idée de progrès dans la pensée de Renan, Paris, Nizet, 1970, Nathalie Richard, "Analogies naturalistes: Taine et Renan», Espaces Temps, 84-85-86, 2004, p. 76-90 et mon article, «L'“éternel fieri" : métamorphoses et histoire dans l'œuvre de Renan ", dans Juliette Azoulai, Azélie Fayolle, Gisèle Séginger (dir.), Les métamorphoses, entre fiction et notion. Littérature et sciences (XVI ${ }^{e}-\mathrm{XXI}^{e}$ siècles), LISAA éditeur, "Savoirs en Textes », p. 125-138, En ligne : https://books.openedition.org/lisaa/1067.

11. Voir Jean Piveteau, «Le débat entre Cuvier et Geoffroy Saint-Hilaire sur l'unité de plan de composition », Revue d'histoire des sciences et de leurs applications, 1950, vol. III, 4, p. 343-363.

12. Renan n'est a priori pas un lecteur de première main des textes scientifiques de Geoffroy Saint-Hilaire, mais il cite avec enthousiasme dans la recension qu'il consacre au cosmos d'Alexander von Humboldt (La Liberté de penser, 1848, non repris dans les volumes ou les œuvres complètes) la biographie présentant ses travaux, rédigée par son fils, Vie, travaux et doctrine scientifique d'Étienne Geoffroy Saint-Hilaire (Paris, Strasbourg, P. Bertrand, Veuve Levrault, 1847).

13. Ernest Renan, L'Avenir de la science, Euvres complètes, t. III, p. 858-859. Le passage est repris in extenso dans l'Histoire générale et système comparé des langues sémitiques (1855).

14. Voir Annie Petit, «Ernest Renan, militant des humanités", Études renaniennes, $n^{\circ} 69$, $3^{\text {e }}$ trimestre 1987, p. 3-32.

15. L'Avenir de la science, op. cit., p. 859.

16. La récapitulation est une notion de rhétorique et de théologie avant d'être une notion de biologie, voir l'article de Jean Céard, "L'analogie du microcosme et du macrocosme », dans Paul Mengal (dir.), Histoire du concept de récapitulation. Ontogenèse et phylogenèse en biologie et sciences humaines, Paris, Milan, Barcelone, Bonn, Masson, 1993, p. 5-15.

17. Ernest Renan, Jean Pommier (éd.), Travaux de jeunesse. 1843-1844, Paris, Les Belles-Lettres, 1931, p. 51.

18. Le verset de la Vulgate est bien métaphorique et non littéral : Ego autem sum vermis et non homo obprobrium hominum et abiectio plebis, soit «Et moi, je suis un ver et non un homme, l'opprobre des hommes et le méprisé du peuple » dans la traduction de Louis Segond. 
19. Le substantif n'apparaît que dans un emploi rare et technique au moment de la rédaction de ces lignes.

20. L'embryon peut ainsi être convoqué pour questionner la notion de substance et sa permanence au fil du temps, sans autre référence christique, voir Cahiers de jeunesse [1845-1846], t. IX, p. 23-24.

21. Ernest Renan, De l'Origine du langage, Euvres complètes, t. viII, p. 107.

22. Voir Judith Schlanger, Les Métaphores de l'organisme, 1971, Paris, L'Harmattan, « Histoire des sciences humaines », 1995.

23. Alain Rey (dir.), « Germe », Dictionnaire historique de la langue française, Paris, Dictionnaires Le Robert, 2010, p. 935.

24. Ernest Renan, Les sciences de la nature et les sciences historiques. Lettre à M. Berthelot, CEuvres complètes, t. I, p. 644.

25. Renan affirme également l'unité biologique de la race humaine, tout en pouvant lui supposer des origines multiples (qui ne recoupent pas les différentes souches originaires des langues). C'est avec son discours Qu'est-ce qu'une nation? de 1882 qu'il prend explicitement ses distances avec le polygénisme biologique, voir Claude Blanckaert, «Les deux parties du problème : Renan et l'ethnographie ", Études renaniennes, $2^{\mathrm{e}}$ trimestre 1994, n 98, p. 62-89.

26. Ce sont des histoires d'abord intellectuelles: Averroès et l'averroïsme est une histoire de la libre-pensée en Occident, cachée sous le nom d'Averroès, quand l'Histoire de l'étude de la langue grecque relate la pratique de la langue grecque, et donc l'érudition, durant tout le Moyen-Âge occidental.

27. Max Black, Models and Metaphors. Studies in Language and Philosophy, Ithaca, New York, Cornell University Press, 1962.

28. Ernest Renan, Vie de Jésus, Cuvres complètes, vol. IV, p. 158-159.

29. Matthieu, 13, 31-32 (traduction de Louis Segond).

30. Voir Gilbert Guisan, Renan et l'art d'écrire, Genève, Droz, 1962.

31. Voir mon article «Renan caricaturiste», L'image, le monstre et l'histoire», Études renaniennes, Brest, $\mathrm{n}^{\circ} 118-119$, p. 109-134.

32. L'Histoire générale et système comparé des langues sémitiques (1855) reste un ouvrage spécialiste, De l'Origine du langage (1848) un opuscule, L'Avenir de la science n'est publié qu'en 1890. L'Histoire des origines du christianisme ne contient pas de telles explications théoriques.

33. Il s'inscrit dans le sillage de David Friedrich Strauss et de La Vie de Jésus ou Examen critique de son histoire (1835), traduit en 1839 et 1853 par Émile Littré, tout en accordant une plus grande place à l'individualité et à la psychologie du Christ.

34. La Vie de Jésus reste à sa sortie un ouvrage onéreux; il faut attendre 1864 pour voir la parution de Jésus, édition populaire (mais aussi remaniée et moins subversive).

35. Ibid. p. 80.

36. Vie de Jésus, op. cit., p. 127 et passim.

37. Voir Gilbert Guisan, Ernest Renan et l'art d'écrire, op. cit., 1962, Gilles Philippe, « Ernest Renan et le rêve du style parfait ", Le XIX siècle et ses langues, Paris, Fondation Singer Polignac et Institut du Monde Arabe, [en ligne], 2012. URL: http://etudes-romantiques.ish-lyon.cnrs.fr/wa_files/ Langues-Philippe.pdf

38. Ibid., p. 81. 


\section{RÉSUMÉS}

Le projet renanien d'une embryogénie de l'esprit humain trouve sa réalisation la plus aboutie dans les deux sommes historiques que sont l'Histoire des origines du christianisme et l'Histoire du peuple d'Israël. Il s'agit pour Renan de remonter aux origines des religions et des langues, en les pensant non seulement selon un modèle organiciste, mais elles-mêmes comme des objets naturels, les produits de l'esprit humain obéissant pour lui aux mêmes lois de nature. Ce projet historien trouve un débouché stylistique dans une imagerie naturaliste qui structure l'ensemble de ses textes. Le projet d'une embryogénie de l'esprit humain trouve alors sa dernière actualisation dans la réalisation d'un texte lui-même pensé comme organisme.

With the History of the Origins of Christianism and the History of the People of Israel, Ernest Renan is able to realize his project of an embryogeny of human spirit. It means for Renan to go up to the origins of religion and language, considered as products of the human spirit which obey the natural laws. This historian project is carried out with a biological inspiration, which innervates all of his texts. This project of an embryogeny of human spirit means the historic text is considered as an organism.

\section{INDEX}

Mots-clés : Renan (Ernest), style, stylistique, métaphore naturaliste, sciences naturelles

Keywords : Renan (Ernest), style, stylistic, naturalistic metaphor, biology

\section{AUTEUR}

\section{AZÉLIE FAYOLLE}

LISAA, Université Gustave-Eiffel 